\title{
Organic Acid Profiling Analysis in Culture Media of Lactic Acid Bacteria by Gas Chromatography-Mass Spectrometry
}

\author{
Jae Yeon Lee ${ }^{\mathrm{a}, \uparrow}$, Duc-Toan Nguyen ${ }^{\mathrm{b}, \uparrow}$, Young Shik Park ${ }^{\mathrm{a}}$, Kyo Yeol Hwang ${ }^{\mathrm{a}}$, Yong Seok Cho ${ }^{\mathrm{a}}$, \\ Kyung-Don Kang ${ }^{\mathrm{a}}$, Jaehwan Yoon ${ }^{\mathrm{b}}$, Jundong Yu ${ }^{\mathrm{c}}$, Sung-Tae Yee ${ }^{\mathrm{d}}$, Young Hwan Ahn ${ }^{\mathrm{e}}$, Gwang Lee, \\ Su-Il Seong ${ }^{\mathrm{ag}, \mathrm{g}, \mathrm{t}}$ and Man-Jeong Paik ${ }^{\mathrm{d}, *}$ \\ ${ }^{a} R \& D$ Center for Life Science, Biotopia Co., Ltd., Chuncheon, Gangwon-do 200-883, Korea \\ ${ }^{b}$ Department of Molecular Science and Technology, Ajou University, Suwon 443-721, Korea \\ ${ }^{c}$ Racing Laboratory, Korea Racing Authority, Gwacheon 427-711, Korea \\ ${ }^{d}$ College of Pharmacy, Sunchon National University, Suncheon, 540-950, Korea \\ ${ }^{e}$ Department of Neurosurgery, Ajou University School of Medicine, Suwon 443-721, Korea \\ ${ }^{f}$ Institute for Medical Science, School of Medicine, Ajou University, Suwon 443-721,Korea \\ ${ }^{g}$ Department of Life Science, The University of Suwon, Hwaseong, Gyeonggi-do 445-743, Korea
}

Received May 30, 2012; Revised June 29, 2012; Accepted August 20, 2012

First published on the web September 20, 2012; DOI: 10.5478/MSL.2012.3.3.74

\begin{abstract}
Organic acid (OA) profiling analysis was performed in culture media from Lactobacillus pentosus $\mathrm{K} 34$ (L. pentosus K34) and Pediococcus lolli PL24 (P. lolli PL24) by gas chromatography-mass spectrometry (GC-MS) following methoxime/ tert-butyldimethylsilyl derivatives. $12 \mathrm{OAs}$ were positively identified in culture media. Most of OA levels from L. pentosus K34 of hetero lactic fermentation were found to be higher when compared with those from P. lolli PL24 of homo lactic fermentation, which may explain different OA metabolism in each strain. In addition, the distorted dodecagonal star patterns were readily distinguishable, and the characteristics of each strain were well represented. The present study demonstrates that the OA metabolic profiling method by GC-MS combined with star pattern recognition is useful for the monitoring study of characteristic OA metabolism in various microorganisms.
\end{abstract}

Key words: Organic acid, Profiling analysis, Lactobacillus pentosus K34, Pediococcus lolli PL24, GC-MS

\section{Introduction}

Organic acids (OAs) were well known as the final products in the metabolic process, which therefore supply information for altered biochemical metabolism from various biological samples including microorganisms. ${ }^{1-3}$ In particular, lactic acid in industrial chemistry fields was reported as important metabolite in fermentation step..$^{4-9}$ In our previous report, ${ }^{10}$ phenyllactic acid was produced in high abundance with lactic acid and acetic acid. Phenyllactic acid is a novel antimicrobial compound active against Gram-positive and Gram-negative bacteria. ${ }^{11,12}$ Phenyllactic acid can be converted from phenylpyruvic acid by NADH dependent lactic acid dehydrogenase (LDH, EC 1.1.1.2.7). ${ }^{14}$ Therefore, the analysis of OAs in culture media and cells of microorganisms is important in the monitoring and screening of microbial availability. In our previous reports, OA profiling analyses by gas chromatography (GC) and GC-mass spectrometry (GC-MS) were found to be useful for

*Reprint requests to Dr. Man-Jeong Paik

E-mail: paik815@sunchon.ac.kr

${ }^{\#}$ Co-corresponding author

'These authors contributed equally to this study the comparative analysis between control and experimental groups. ${ }^{9,10,14,15}$

Gram positive Lactobacillus pentosus K34 (L. pentosus $\mathrm{K} 34)$ is facultative anaerobic and hetero-fermentative as rod-shaped lactic acid bacterium with rounded ends, which may occur singly, in pairs or short chains. It is nonmotile and grows at $10{ }^{\circ} \mathrm{C}$ and $40{ }^{\circ} \mathrm{C}$ but not at $45{ }^{\circ} \mathrm{C} .{ }^{16} \mathrm{In}$ contrast, Pediococcus lolli PL24 (P. lolli PL24) is homo-fermentative Gram-positive lactic acid bacterium. It is coccus-shaped, nonspore-forming, nonmotile, and occurs in pairs or tetrads. ${ }^{17}$ $L$. pentosus $\mathrm{K} 34$ and $P$. lolli PL24 have two different characteristics. First, P. lolli PL24 has a thermo-tolerance and grows at high temperature of $47^{\circ} \mathrm{C}$, while $L$. pentosus $\mathrm{K} 34$ cannot grow at high temperature of $47^{\circ} \mathrm{C}$. Thermotolerant lactic acid bacteria has a beneficial probiotic advantage, for example, maintaining cell viability during manufacturing processes such as heat drying. ${ }^{18}$ Second, $P$. lolli PL24 has been known to be obligately homofermentative, while $L$. pentosus has been known to be facultatively hetero-fermenative. Normally, the metabolic pathway of homo lactic fermentation uses the EmbdenMeyerhoff-Parnas (EMP) pathway and the metabolic pathway of hetero lactic fermentation uses the pentose phosphate pathway. Thus, in this study, our previous OA 
profiling method ${ }^{9,14,15}$ using methoxime/tert-butyldimethylsilyl (MO/TBDMS) derivatives in combination with GCMS was applied to culture media of L. pentosus $\mathrm{K} 34$ and $P$. lolli PL24 for the monitoring of altered OA metabolism.

\section{Experimental Section}

\section{Strain and culture condition}

L. pentosus $\mathrm{K} 34$ with the strong inhibitory activity against gastro-intestinal pathogenic bacteria was isolated from the small intestine of Korean native chicken ${ }^{10,11}$ and P. lolli PL24 with the inhibitory activity against gastrointestinal pathogenic bacteria as well as thermo-tolerance at $47^{\circ} \mathrm{C}$ was newly isolated from the waste of milk processing $(n=3)$. Each strain was activated at $37^{\circ} \mathrm{C}$ for 2 days on de Man, Rogosa and Sharpe (MRS) (BD, USA) agar plate $(n=3)$. Then one loopful of each grown single colony was suspended into $1 \mathrm{~mL} 0.85 \% \mathrm{NaCl}$ solution. Using syringe, $300 \mu \mathrm{L}$ of each cell suspension was inoculated into $30 \mathrm{~mL}$ glass vial sealed by rubber and aluminum cap containing $20 \mathrm{~mL}$ MRS broth and incubated at $37{ }^{\circ} \mathrm{C}$ for 3 days without shaking. After cultivation, the culture broth was centrifuged at $10,000 \mathrm{~g}$ for $10 \mathrm{~min}$. The supernatant was taken to the fresh new conical tube (SPL, Korea) and stored at $-70{ }^{\circ} \mathrm{C}$ deep freezer (Ilsin, Korea).

\section{Chemicals and reagents}

OA standards including 3,4-dimethoxybenzoic acid as internal standard (IS), triethylamine (TEA) methoxyamine hydrochloride were purchased from Sigma-Aldrich. $\mathrm{N}$ Methyl- $N$-(tert-butyldimethylsilyl)trifluoroacetamid (MTBSTFA) $+1 \%$ tert-butyldimethylchlorosilane was provided from Thermo Scientific (Bellefonte, PA, USA). Toluene, diethyl ether, ethyl acetate, and sodium chloride (pesticide grade) were obtained from Kanto Chemical (Chuo-ku, Tokyo, Japan). Sulfuric acid was purchased from Samchun pure chemical Co. Ltd. (Pyeongtaek, Gyeonggido, South Korea). All other chemicals were of analytical reagent grade.

\section{Gas chromatography-mass spectrometry}

Derivatized samples were analyzed using an Agilent $6890 \mathrm{~N}$ gas chromatograph interfaced to an Agilent 5975B mass-selective detector ( $70 \mathrm{eV}$, electron ionization source). The mass spectra were scanned in the mass range of 50$650 \mathrm{u}$ at a rate of $0.99 \mathrm{scans} / \mathrm{s}$. The temperatures of the injector, interface, and ion source were 260, 300, and $230{ }^{\circ} \mathrm{C}$, respectively. An Ultra-2, cross-linked capillary column coated with $5 \%$ phenyl-95\% methylpolysiloxane bonded phase $(25 \mathrm{~m} \times 0.20 \mathrm{~mm}$ I.D., $0.11 \mathrm{~mm}$ film thickness, Agilent Technologies, Santa Clara, CA, USA) was used for all analyses. Helium was used as the carrier gas at a flow rate of $0.5 \mathrm{~mL} / \mathrm{min}$ in the constant flow mode. Samples $(1 \mu \mathrm{L})$ were introduced in split-injection mode $(10: 1)$, and the oven temperature was set initially at $100{ }^{\circ} \mathrm{C}(2 \mathrm{~min})$, then increased to $250{ }^{\circ} \mathrm{C}$ at rate of $5^{\circ} \mathrm{C} / \mathrm{min}$ and finally programmed to $300{ }^{\circ} \mathrm{C}$ at rate of $20{ }^{\circ} \mathrm{C} / \mathrm{min}(5 \mathrm{~min})$.

\section{Sample preparation for measurements of organic acids in cell culture media}

Control media (MRS), and culture media from L. pentosus K34 and P. lolli PL24 were used for experiments $(\mathrm{n}=3)$. Aliquots of culture media $(20 \mu \mathrm{L})$ containing IS $(5 \mu \mathrm{g})$ were spiked to distilled water $(1 \mathrm{~mL})$ and reacted with methylhydroxylamine hydrochloride $(1 \mathrm{mg})$ in alkaline condition at $60{ }^{\circ} \mathrm{C}$ for $30 \mathrm{~min}$ for conversion into $\mathrm{MO}$ derivative. The reaction mixture was then acidified to $\mathrm{pH}<2$ with $10 \%$ sulfuric acid solution, saturated with sodium chloride, and extracted with diethyl ether $(4 \mathrm{~mL})$ followed by ethyl acetate $(2 \mathrm{~mL})$. After addition of TEA $(5 \mu \mathrm{L})$, the combined extracts were evaporated under a gentle stream of nitrogen $\left(40{ }^{\circ} \mathrm{C}\right)$ to dryness. Toluene $(20 \mu \mathrm{L})$ as the solvent and MTBSTFA $(20 \mu \mathrm{L})$ as the silylation reagent were added to the residue, and the mixture was heated at $60{ }^{\circ} \mathrm{C}$ for $30 \mathrm{~min}$ to form MO/TBDMS derivatives prior to analysis by GC-MS.

\section{Star symbol plotting}

The mean peak area ratios to IS of OAs confirmed in the control media and culture media from L. pentosus K34 and P. lolli PL24 were normalized to the corresponding mean of those in the L. pentosus K34. Then normalized levels of 12 OAs were plotted with lines radiating for star symbol plotting using Microsoft Excel (Microsoft, Redmond, WA), as described in previous report. ${ }^{19}$

\section{Results and Discussion}

GC-MS profiles from the control media (A), L. pentosus K34 (B), and P. lolli PL24 (C) are showed in Figure 1. Seven OAs were screened in the control media (A), while 12 and 10 OAs were identified in L. pentosus K34 (B) and P. lolli PL24 (C), respectively (see also Table 1). In control media, lactic acid (No.2) was most abundant, followed by oleic acid (No.9), pyruvic acid (No.1), glycolic acid (No.3), succinic acid (No.7), and citric acid (No.12). Lactic acid (No.2) in L. pentosus K34 was most abundant, followed by oleic acid (No.9), succinic acid (No.7), pyruvic acid (No.1) and phenyllactic acid (No.8). In contrast, in P. lolli PL24, lactic acid (No.2) was most abundant, followed by oleic acid (No.9), pyruvic acid (No.1), phenyllactic acid (No.8), and succinic acid (No.7). The overall OA levels of $L$. pentosus K34 were higher when compared with those of the $P$. lolli PL24. This finding may suggest that OAs produced by P. lolli PL24 was smaller than those of L. pentosus K34. In particular, $P$. loll PL24 mainly produced lactic acid (No.2) and phenyllactic acid (No.8) which are assumed to be produced from pyruvic acid and phenylpyruvic acid by $\mathrm{LDH}$ via EMP pathway. The difference in the overall OA levels is due to the different metabolic pathway of each 
Table 1. Values of organic acids in control media, and cultured media with L. pentosus K34 and P. lolli PL24.

\begin{tabular}{|c|c|c|c|c|c|c|c|}
\hline \multirow{2}{*}{ No. } & \multirow{2}{*}{ Organic acid } & \multicolumn{3}{|c|}{ Peak area ratio $($ mean $\pm S D, n=3)$} & \multicolumn{3}{|c|}{$\begin{array}{c}\text { Normalized values }^{\mathrm{b}} \\
\end{array}$} \\
\hline & & \multicolumn{2}{|c|}{ Control media L. pentosus K34 } & \multirow{2}{*}{$\begin{array}{c}\quad \text { P. lolli } \text { PL24 } \\
0.397 \pm 0.015\end{array}$} & \multicolumn{2}{|c|}{ Culture media L. pentosus K34 } & \multirow{2}{*}{$\begin{array}{c}\text { P. lolli PL24 } \\
1.12\end{array}$} \\
\hline 1 & Pyruvic acid & $0.420 \pm 0.066$ & $0.355 \pm 0.013$ & & 1.18 & 1.00 & \\
\hline 2 & Lactic acid & $1.081 \pm 0.181$ & $23.840 \pm 1.008$ & $21.363 \pm 0.752$ & 0.05 & 1.00 & 0.90 \\
\hline 3 & Glycolic acid & $0.404 \pm 0.012$ & $0.102 \pm 0.007$ & $0.116 \pm 0.004$ & 3.96 & 1.00 & 1.14 \\
\hline 4 & 2-Hydroxyisovaleric acid & N.D. ${ }^{\mathrm{a}}$ & $0.038 \pm 0.002$ & N.D. & 0.00 & 1.00 & 0.00 \\
\hline 5 & 2-Hydroxyisocaproic acid & N.D. & $0.159 \pm 0.009$ & $0.012 \pm<0.001$ & 0.00 & 1.00 & 0.08 \\
\hline 6 & 2-Hydroxy-3-methylvaleric acic & d N.D. & $0.031 \pm 0.002$ & N.D. & 0.00 & 1.00 & 0.00 \\
\hline 7 & Succinic acid & $0.213 \pm 0.013$ & $0.430 \pm 0.025$ & $0.247 \pm 0.005$ & 0.49 & 1.00 & 0.57 \\
\hline 8 & Phenyllactic acid & $0.001 \pm<0.001$ & $0.164 \pm 0.009$ & $0.353 \pm 0.011$ & 0.00 & 1.00 & 2.16 \\
\hline 9 & Oleic acid & $0.881 \pm 0.076$ & $0.856 \pm 0.058$ & $0.808 \pm 0.039$ & 1.03 & 1.00 & 0.94 \\
\hline 10 & 4-Hydroxyphenyllactic acid & N.D. & $0.108 \pm 0.007$ & $0.162 \pm 0.006$ & 0.00 & 1.00 & 1.49 \\
\hline 11 & Indole-3-lactic acid & N.D. & $0.005 \pm 0.001$ & $0.008 \pm<0.001$ & 0.00 & 1.00 & 1.51 \\
\hline 12 & Citric acid & $0.155 \pm 0.015$ & $0.019 \pm 0.002$ & $0.053 \pm 0.003$ & 8.20 & 1.00 & 2.82 \\
\hline
\end{tabular}

${ }^{\mathrm{a}}$ Not determined

${ }^{\mathrm{b}}$ Values normalized to corresponding mean values of organic acids in cultured media with $L$. pentosus $\mathrm{K} 34$. IS = 3,4-Dimethoxybenzoic acid
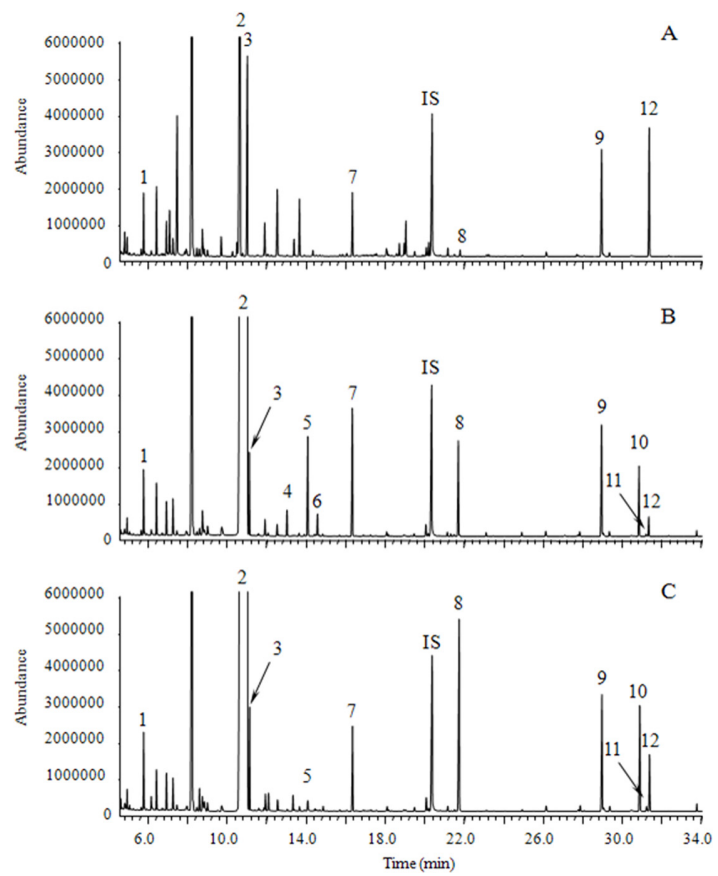

Figure 1. Organic acid profiles as methoxime/tert-butyldimethylsilyl derivatives of control media (A), culture media from $L$. pentosus $\mathrm{K} 34$ (B) and P. lolli PL24 (C). 1 = Pyruvic acid, $2=$ Lactic acid, 3 = Glycolic acid, $4=2$-Hydroxyisovaleric acid, $5=$ 2-Hydroxyisocaproic acid, 6=2-Hydroxy-3-methylvaleric acid, $7=$ Succinic acid, $8=$ Phenyllactic acid, $9=$ Oleic acid, $10=4-$ Hydroxyphenyllactic acid, 11 = Indole-3-lactic acid, 12 = Citric acid, IS = 3,4-Dimethoxybenzoic acid.

strain. P. lolli PL24 with homo lactic fermentation mainly produced lactic acid from pyruvic acid by $\mathrm{LDH}$, while L. pentosus K34 with hetero lactic fermentation produced
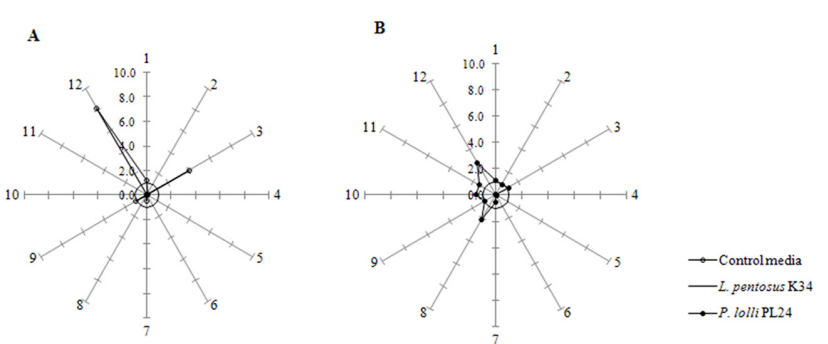

Figure 2. Star symbol plots of control media, L. pentosus K34, and $P$. lolli PL24 based on the mean levels of the 12 OAs obtained through normalization to the corresponding mean values of $L$. pentosus K34. (A) L. pentosus K34 vs. control and (B) L. pentosus K34 vs. P. lolli PL24. The numbers on the rays correspond to those in Table 1.

the various OAs including lactic acid throughout the different metabolic pathway. Typically, hydroxyl acids such as No.4, No.5, and No.6 produced by L. pentosus K34 might be produced by central metabolism and/or amino acid metabolism. Specifically, $\alpha$-keto acids were produced by biosynthetic enzymes involved in the central metabolism and/ or also by aminotransferases from amino acids. Subsequently, hydroxyl acids were produced by hydroxyl acid dehydrogenases from $\alpha$-keto acids. ${ }^{20}$

The OA levels in the media of control and P. lolli PL24 were normalized to the corresponding mean OA levels in the media of L. pentosus K34 (Table 1), which proved very informative by expressing the alteration (ranging from 0.00 to 8.20) of the OA values. In control media, the levels of glycolic acid (No.3) and citric acid (No.12) were highly elevated compared to those of L. pentosus K34 and P. lolli PL24. This is because these two OAs are used for growth of L. pentosus K34 and P. lolli PL24. In P. lolli PL24, the 
abundances of phenyllactic acid (No.8), 4-hydroxyphenyllactic acid (No.10), indole-3-lactic acid (No. 11), and citric acid (No.12) in the $P$. lolli PL24 were much larger, while the abundances of 2-hydroxyisovaleric acid (No.4), 2hydroxyisocaproic acid (No.5), 2-hydroxy-3-methylvaleric acid (No.6), and succinic acid (No.7) were smaller than those of the L. pentosus K34. The star symbol plots drawn based on these values displays distorted patterns (Figure 2) for the control media (Figure 2A) and the P. lolli PL24 (Figure 2B) when compared to the pattern of the $L$. pentosus K34. Thus, this result may explain different OA metabolism and fermentation process of L. pentosus K34 and P. lolli PL24.

\section{Conclusions}

OA metabolic profiles in cell growth media from $L$. pentosus $\mathrm{K} 34$ as hetero fermentative and P. lolli PL24 as homo fermentative showed different patterns. And the star patterns were readily distinguishable and characteristic of each strain. This may explain for usefulness of OA profiling method using GC-MS combined with star pattern recognition for monitoring of altered and characteristic OA metabolism in various microorganisms.

\section{Acknowledgements}

This research was supported by the Basic Science Research Program through the National Research Foundation of Korea funded by the Ministry of Education, Science, and Technology (2010-0021443) to M. J. Paik and (2010-0023846). G. Lee. And the Priority Research Centers Program through the National Research Foundation of Korea (NRF) funded by the Ministry of Education, Science and Technology (20100028294).

\section{References}

1. Matsumoto, I.; Kuhara, T. Mass Spectrom. Rev. 1987, 6, 77.

2. Liebich, H. M.; Forst, C. J. Chromatogr. 1990, 525, 1.
3. Duez, P.; Kumps, A.; Mardens, Y. Clin. Chem. 1996, 42, 1609.

4. Caligiani, A.; Cirlini, M.; Palla, G.; Ravaglia, R.; Arlorio, M. Chirality 2007, 19, 329.

5. Grabar, T. B.; Zhou, S.; Shanmugam, K. T.; Yomano, L. P.; Ingram, L. O. Biotechnol. Lett. 2006, 28, 1527.

6. Joshi, D. S.; Singhvi, M. S.; Khire, J. M.; Gokhale, D. V. Biotechnol. Lett. 2010, 32, 517.

7. John, R. P.; Nampoothiri, K. M.; Pandey, A. Appl. Microbiol. Biotechnol. 2007, 74, 524.

8. Ewaschuk, J. B.; Zello, G. A.; Naylor, J. M.; Brocks, D. R. J. Chromatogr. B 2002, 781, 39.

9. Paik, M. J.; Nguyen, D. T.; Yoon, J.; Chae, H. S.; Kim, K. R.; Lee, G.; Lee, P. C. Bull. Korean Chem. Soc. 2011, 32, 2418.

10. Lee, J. Y.; Hwang, K. Y.; Kim, K.; Seong, S. I.; Park, Y. S.; Paik, M. J.; Kim, K.R. Kor. J. Microbiol. Biotechnol. 2002, 30, 241.

11. Dieuleveux, V.; van der Pyl, D.; Chataud, J.; Gueguen, M. Appl. Environ. Microbiol. 1998, 64, 800.

12. Dieuleveux, V.; Lemarinier, S. ; Gueguen, M. Intl. J. Food Microbiol. 1998, 40, 177.

13. Li, X. F.; Jiang, B.; Pan, B. L.; Mu, W. M. ; Zhang, T. J. Agric. Food Chem. 2008, 56, 2392.

14. Paik, M. J.; Kim, K. R. J. Chromatogr. A 2004, 1034, 13.

15. Paik, M. J.; Cho, E. Y.; Kim, H.; Kim, K. R.; Choi, S.; Ahn, Y. H.; Lee, G. Biomed. Chromatogr. 2008. 22, 450.

16. Zanoni, P.; Farrow, J. A. E.; Phillps, B. A.; Collins, M. D. Intl. J. Systematic Bacteriol. 1987, 37, 339.

17. Doi, K.; Nishizaki, Y.; Fujino, Y.; Ohshima, T; Ohmomo, S.; Ogata, S. Intl. J. Systematic and Evolutionary Microbiol. 2009, 59, 1007.

18. Ross, R. P.; Desmond, C.; Fitzgerald, G. F.; Stanton, C. J. Appl. Microbiol. 2005, 98, 1410.

19. Paik, M. J.; Li, W. Y.; Ahn, Y. H.; Lee, P. H.; Choi, S.; Kim, K. R.; Kim, Y. M.; Bang, O. Y.; Lee, G. Clin. Chim. Acta 2009, 402, 25.

20. van Kranenberg, R.; Leerebezem, M.; Vlieg, J. van H.; Ursing, B.M.; Boekhorst, J.; Smit, B. A.; Ayad, E. H. E.; Smit, G.; Siezen, R. J. Intl. Dairy J. 2002, 12, 111. 